\section{Military Technical College Kobry El-kobbah, Cairo, Egypt}

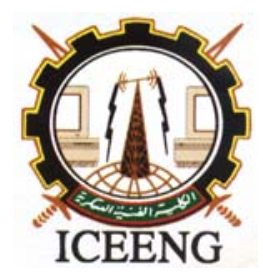

\section{$5^{\text {th }}$ International Conference on Electrical Engineering}

I. ICEENG 2006

\title{
An Integrated Approach for Probabilistic Assessment of Ionized Fields of HVDC Lines
}

\author{
Mohamed M. Abouelsaad $^{1}$, Ibrahim M. El Shair ${ }^{2}$ and Ihab F. Ghariany ${ }^{3}$
}

\begin{abstract}
An integrated statistical approach, based on Monte Carlo simulation, is developed in this paper for probabilistic exposure assessment under HVDC transmission lines. The validity and adequacy of traditional, deterministic, models for HVDC ionized fields have been repeatedly questioned in literature. The present work recognizes the probabilistic nature of the exposure variables, electric field intensity and ion current density, and caters for their inherent uncertainties in order to simulate the realistic dc line environment . Randomness in the factors influencing the ionized field quantities is identified and accounted for, namely, the wind speed, the line sag, the corona onset gradient, and the location of objects under the line. Based on an overall probabilistic approach, the integrated influence of these factors on the exposure parameters is evaluated and assessed.
\end{abstract}

Keywords: Probabilistic methods, DC ionized fields, uncertainties.

\section{INTRODUCTION}

With the growing need for electric energy, direct current has been, recently, established as an attractive economical and operational alternative for transmitting large bulks of electric power over extra long distances [1-4]. The ion-flow electrification phenomenon, caused by ionic conduction through the air and associated with dc power transmission lines, usually characterizes the potential biological impact of exposure to dc ionized fields [5-7]. Two fundamental parameters; the electric field and ion current density, are denoted in this regard $[5,6]$. Both parameters are known to be functions of several factors, namely, the wind speed, the line sag which in turn determines the line height, the corona onset gradient. In addition, the random location of an object near a dc power line subjects it to random magnitudes of prevailing electric field and current density in the dc line environment[2,3].

It is quite difficult to separate the effect of the above factors under HVDC lines, in outdoor studies, because their random changes are nearly accompanied by changes in the electric field and current density profiles under the lines [1-3,5]. Traditional assessment of the dc ionized field exposure problem may lead to the application of relatively large safety factors. This is in order to cover the uncertainties associated with the influencing factors. Such determining factors can; thus, be treated as random quantities through probability based techniques. The paper presents an overall probabilistic approach through which the integrated influence of these factors, on ionized field quantities, is evaluated and assessed.

\footnotetext{
${ }^{1}$ Associate professor, Faculty of Engineering at Shoubra, Benha University, Shoubra , Cairo, Egypt

${ }^{2}$ Professor, Faculty of Engineering, Ain-Shams University, Cairo, Egypt

${ }^{3}$ Graduate student, Ministry of the Interior, Cairo, Egypt
} 


\section{METHOD OF ANALYSIS}

A unipolar ionized field is described by the following set of equations,

$$
\begin{aligned}
\nabla . \mathbf{E} & =\rho / \varepsilon \\
\mathbf{J} & =\rho(k \mathbf{E}+w) \\
\nabla . \mathbf{J} & =0 \\
\mathbf{E} & =\nabla \boldsymbol{\emptyset}
\end{aligned}
$$

where $E$ is the electric field, $\rho$ is the space charge density, $J$ is the current density, $\varepsilon$ is the permittivity of free space, $\mathrm{k}$ is the ion mobility, and $\mathrm{w}$ is the wind speed. The above equations are solved iteratively along each field line using the iterative algorithm given in [8]. The boundary conditions are the potentials on the energized conductor and on the ground, $\varnothing=\mathrm{V}$ and $\varnothing=0$ respectively. The electric field at the surface is assumed constant at the corona onset value. In order to demonstrate the proposed approach, a practical $600 \mathrm{KV}$ dc negative monopolar line with $4 \times 3.05 \mathrm{~cm}$ diameter conductor and $45.7 \mathrm{~cm}$ sub-conductor spacing is used as a model in the present analysis [5,6]. The line height is $12.2 \mathrm{~m}$ at mid span, $30.24 \mathrm{~m}$ at the tower, and the line span is taken equal $450 \mathrm{~m}$ while the edge of the right-of-way (ROW) is 40m (approximately 3.2 times the height). The above equations are solved for this geometry to obtain the electric field $E$ and the current density $J$ at any point in the line neighborhood $(\mathrm{x}, \mathrm{z})$ where $\mathrm{x}$ is the lateral distance measured from the tower centerline, and $\mathrm{z}$ is the longitudinal distance measured from mid-span. Due to the symmetry of the geometry, only the area under the line bounded by $\mathrm{x}=0-40 \mathrm{~m}$ and $\mathrm{z}=0-150 \mathrm{~m}$ is considered.

\section{EFFECT OF UNCERTAINTIES IN THE IONIZED FIELD FACTORS}

In the following sections, the randomness in the ionized field parameters is identified and analyzed. The three main parameters considered are the wind speed $w$, the line sag (or the corresponding line height $H$ ) and the corona onset gradient $G$ which are known to have a significant influence on the ionized field quantities. Each individual factor is modeled with a uniform probability distribution and the governing equations are subsequently solved to obtain the corresponding ionized field quantities.

\subsection{Effect of the Wind Speed}

In order to study the influence of the wind speed, the wind is assumed to blow from left to right normal to the line. The wind speed is assumed to vary randomly, uniform, between 0 and $2.5 \mathrm{~m} / \mathrm{s}$, as the wind influence seems to be more pronounced within this range [5]. It was observed, in an earlier study, that increased magnitudes of electric fields appear in the downwind side of the line $[1,3,5]$. Similar results were obtained for the current density. The frequency of occurrence of an ambient field intensity, E, or ambient current density, J, at any point near the line is obtained by, first, producing the entire $\mathrm{E}$ and $\mathrm{J}$ contour maps; similar to those obtained earlier [3]. The probability density in this case, of a certain $\mathrm{E}$ or $\mathrm{J}$ is the ground area where $\mathrm{E}$ or $\mathrm{J}$ attains that certain value relative the total area of the site. The histograms of ambient field and current density are obtained as shown in Fig. 1 for the electric field and Fig. 2 for the current density. They appeared to follow closely an exponential distribution, and can be shown to take the following form, in case of an E-map in the downwind side,

$$
p(E)=f[E(w)]
$$


It is assumed that,

$$
f[E(w)]=k e^{-\left[E /\left(E_{m}-E_{r}\right)\right]}
$$

where $E$ is the electric field at ground, $w$ is the wind speed, $E_{m}$ is the mean value of the field distribution, $E_{r}$ is the field at the ROW and $k$ is a constant and they are, respectively, derived as follows,

$$
E_{m}=C_{1} W+E_{W 0}
$$

where $E_{W 0}$ is the mean electric field at $w=$ zero and a assuming a linear relationship exists between $E_{m}$ and $w$, then $C_{1}=3.62$ in this case [1]. As it was observed that the minimum value of electrical field at different wind speeds always occur at the edge of the ROW for the wind range considered, then;

$$
E_{r}=C_{2} W+E_{r W 0}
$$

where $E_{r w o}$ is the field at ROW at w = zero, and a linear relationship exists between $E_{r}$ and $w$ , then $C_{2}=3.3$ in this case [1]. The constant $k$ is given as,

$$
k=\left[\left(E_{m}-E_{r}\right) e^{-\left[E /\left(E_{m}-E_{r}\right)\right]}\right]^{-1}
$$

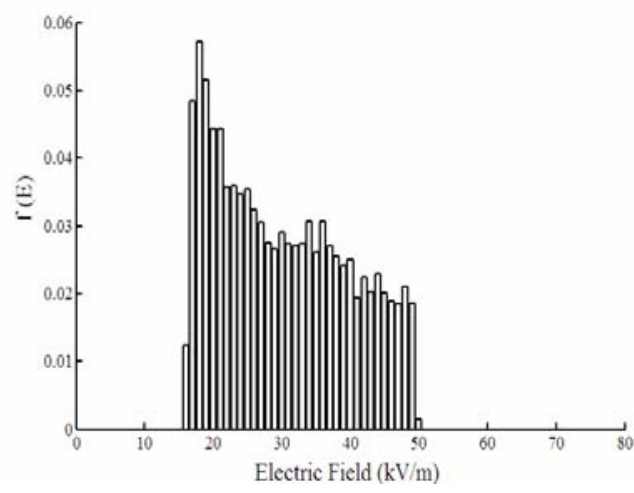

Fig. 1 Histogram of $E$ - field, $w=0-2.5 \mathrm{~m} / \mathrm{s}$, $H=12.2 \mathrm{~m}, G=11.04$

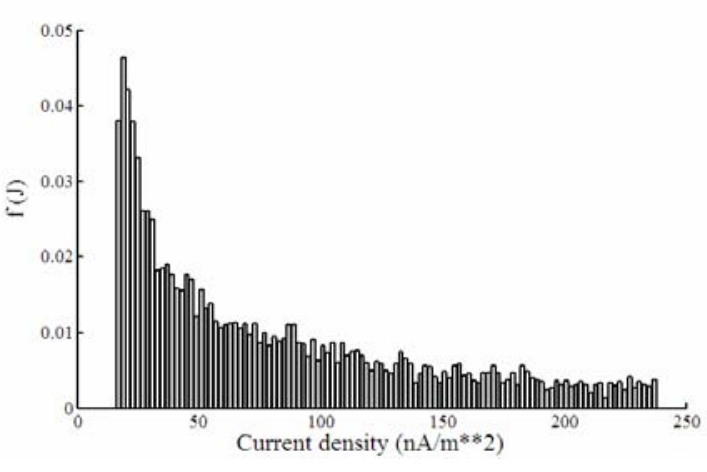

Fig. 2 Histogram of $J$ - field, $w=0-2.5 \mathrm{~m} / \mathrm{s}$, $H=12.2 \mathrm{~m}, G=11.04$

Similar equations were obtained for the current density profiles. Inspection of the above equations shows that with fewer measurements (or calculations) at calm conditions (no wind), the probability of occurrence of a given electric field value ( at a given wind speed) can be easily determined using the above expressions.

\subsection{Effect of the Line Sag}

It has been shown earlier that the conductor height has a significant influence on the electric field, and ion current density under dc power lines [1,7]. In the following, the height is considered to be the only random factor in
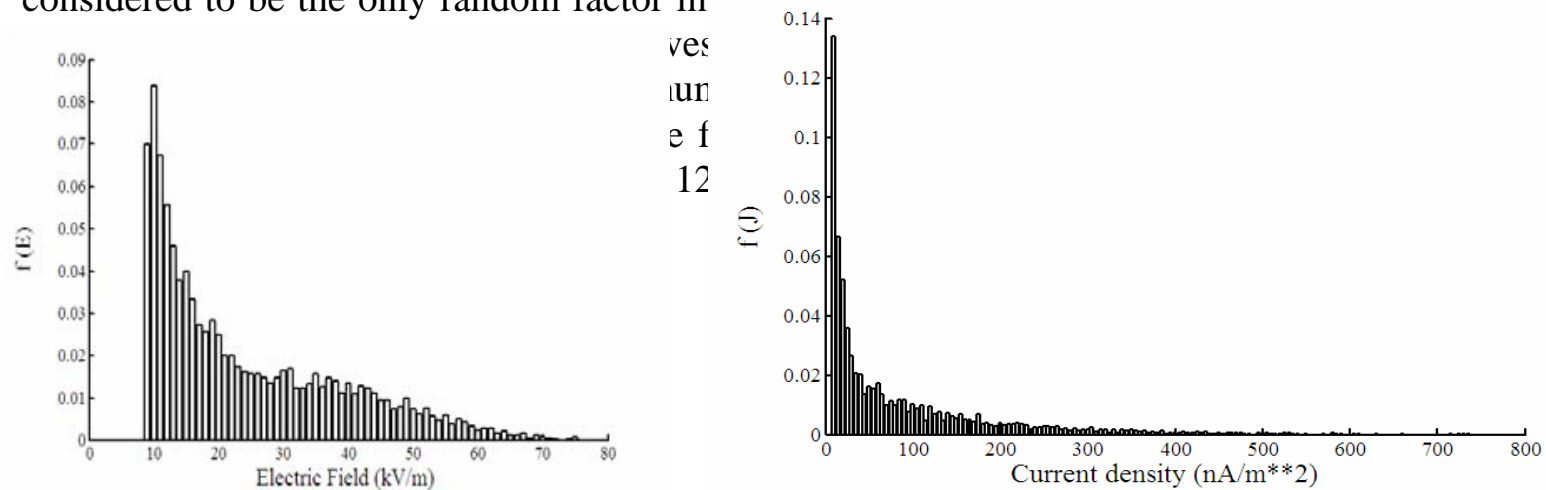
Fig. 3 Histogram of $E$ - field, $H=8.5-12.2 \mathrm{~m}$, $w=0 \mathrm{~m} / \mathrm{s}, G=11.04 \mathrm{kV} / \mathrm{cm}$
Fig. 4 Histogram of $J$ - field, $H=8.5-12.2 \mathrm{~m}$, $w=0 \mathrm{~m} / \mathrm{s}, G=11.04 \mathrm{kV} / \mathrm{cm}$

of electric fields appear by decreasing the height at the mid span, $H$. Similar results were obtained for the current density profiles.

Taking the height, alternatively the sag, as the only factor influencing the frequency of occurrence of $E$ and $J$, and similar to the wind case, the distributions of $E$-map in the downwind side are developed. The histograms of field and current density are obtained as shown in Fig. 3 for the electric field and Fig. 4 for the current density when $\mathrm{H}$ varies randomly. Similar to the wind case, the histograms appeared to follow closely an exponential distribution in the following from,

$$
\begin{gathered}
p(E)=f[E(H)] \\
f[E(H)]=k e^{-\left[E /\left(E_{m}-E_{r}\right)\right]}
\end{gathered}
$$

where $E$ is the electric field at ground, $H$ is the height at the mid span, $E_{m}$ is the mean value, $E_{r}$ is the field at ROW and $k$ is a constant and they are, respectively, given by,

$$
E_{m}=C_{3} H+E_{H 0}
$$

$E_{H O}$ is the mean electric field corresponding to the minimum sag ( which in this case corresponds to $H=12.2 \mathrm{~m}$ ). Assuming a linear relationship exists between $E_{m}$ and $H$, then $C_{3}=-1.029$ in this case [1]. Also, as it was observed that the minimum value of electrical field at different height always occur at the edge of the ROW for the height range considered, then;

$$
E_{r}=C_{4} H+E_{r H 0}
$$

where $E_{r H O}$ is the field at ROW at $H=12.2 \mathrm{~m}$, which corresponds to the minimum sag, and assuming a linear relationship exists between $E_{r}$ and $H$, then $C_{4}=-0.054$ in this case [1]. The constant $k$ is given as,

$$
k=\left[\left(E_{m}-E_{r}\right) e^{-\left[E /\left(E_{m}-E_{r}\right)\right]}\right]^{-1}
$$

Similar equations were obtained for the current density profiles. Again, similar to the wind case, the probability of a given $E$ or $J$ value ( for a given $H$ ) can be easily determined from few measurements or calculations under a normal operation of the line.
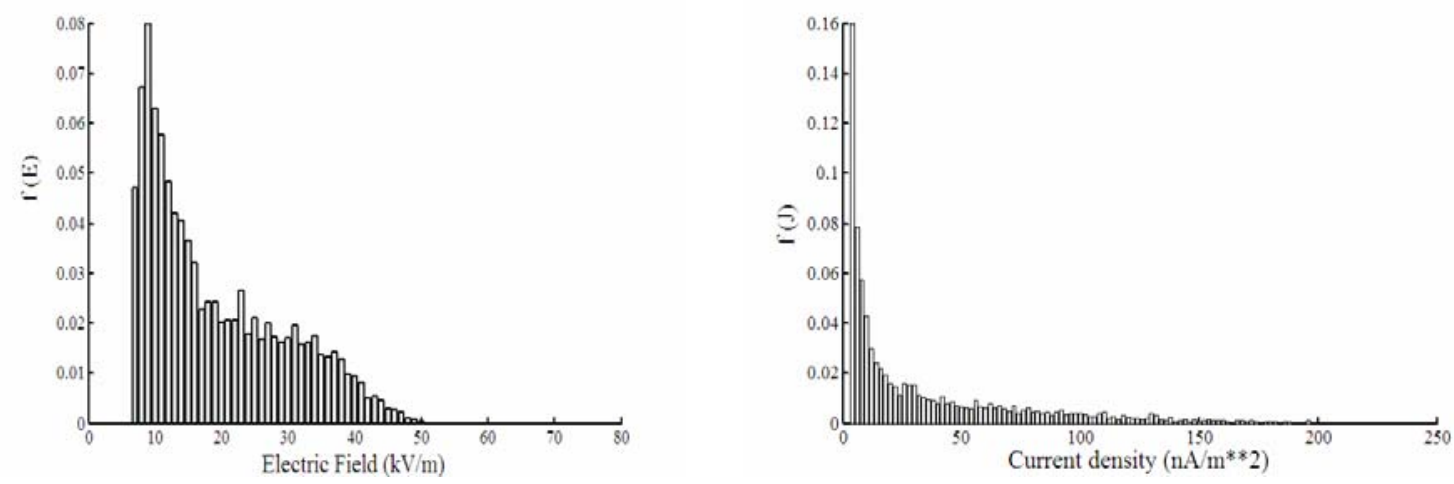
Fig. 5 Histogram of $E$ - field, $G=11.04-16.79 \mathrm{kV} / \mathrm{cm}$, $w=0 \mathrm{~m} / \mathrm{s}, H=12.2 \mathrm{~m}$
Fig. 6 Histogram of $J$ - field, $G=11.04-16.79 \mathrm{kV} / \mathrm{cm}$, $w=0 \mathrm{~m} / \mathrm{s}, H=12.2 \mathrm{~m}$

\subsection{Effect of the Corona Onset Gradient}

Experimental studies on practical dc line geometries showed that the roughness factor $\mathrm{m}$ used to determine the corona onset gradient may vary within the range of 0.3 to 0.5 depending the prevailing weather and the conductor conditions [5,6]. Accordingly, the calculation of the corona onset gradient is carried out using the relation in [8] with m varying randomly uniform between 0.3 and 0.5 . It was shown earlier in [1] that increased magnitudes of electric fields appear by decreasing the corona onset gradient $G$. Similar results were obtained for the current density profiles.

Taking the corona onset gradient as the only random factor influencing the frequency of occurrence of $E$ and $J$, the histograms of ambient field and current density are obtained as shown in Fig. 5 for the electric field and Fig. 6 for the current density. Similar to the wind and height cases, the histograms appeared to follow closely an exponential distribution in the following from,

$$
\begin{gathered}
p(E)=f[E(G)] \\
f[E(G)]=k e^{-\left[E /\left(E_{m}-E_{r}\right)\right]}
\end{gathered}
$$

where $E$ is the electric field at ground, $G$ is the corona onset gradient, $E_{m}$ is the mean value, $E_{r}$ is the field at ROW, and $k$ is constant. , and they are given by,

$$
E_{m}=C_{5} G+E_{G 0}
$$

where, $E_{G 0}$ is the mean electric field at $G=11.04 \mathrm{kV} / \mathrm{cm}$, which is the lowest corona onset gradient considered. Assuming a linear relationship exists between $E_{m}$ and $G$, then $C_{5}=-1.34$ in this case [1]. Also, as it was observe that the minimum value of electrical field at different corona onset gradient always occur at the edge of the ROW for the onset gradient range considered, then;

$$
E_{r}=C_{6} G+E_{r G 0}
$$

where, $E_{r G O}$ is the electric field at $G=11.04 \mathrm{kV} / \mathrm{cm}$, which is the lowest field gradient considered, and assuming a linear relationship exists between $E_{r}$ and $H$, then $C_{6}=-0.639$ in this case [1]. The constant $k$ is given as,

$$
k=\left[\left(E_{m}-E_{r}\right) e^{-\left[E /\left(E_{m}-E_{r}\right)\right]}\right]^{-1}
$$

Similar equations were obtained for the current density profiles. Again, similar to the wind and height cases, the probability of a given $E$ or $J$ value ( for a given $G$ ) can be easily determined from few measurements or calculations under a normal operation of the line.

\subsection{Random Location of Objects Under The Line}

As the presence of the objects under the line is not deterministic, it has been taken uniformly random over the entire power line site, in both the $\mathrm{x}$ and $\mathrm{z}$ directions, in order to determine its influence upon the electric field and current density profiles.

\section{STATISTICAL ASSESSMENT}

In order to assess the joint effect of all previous factors, an algorithm was developed to achieve this purpose. The steps of the algorithm are: 
1-The longitudinal distance from mid span $\mathrm{z}$ is taken uniformly random from 0 to $\mathrm{z}_{\mathrm{f}}$, where $\mathrm{z}_{\mathrm{f}}$ is taken equal to $150 \mathrm{~m}$.

2-The lateral distance $\mathrm{x}$ is taken uniformly random from tower centerline 0 to the edge of ROW, where the edge of ROW is considered equal to $40 \mathrm{~m}$ in the downwind side.

3-The wind speed varies uniformly random from 0 to $2.5 \mathrm{~m} / \mathrm{s}$.

4-The height at the mid span is assumed to vary randomly, uniform, between 8.5 and $12.2 \mathrm{~m}$.

5-The corona onset gradient is assumed to vary uniformly random between 11.04 and 16.79 $\mathrm{KV} / \mathrm{cm}$.

6- Determine the electric field $E$ and current density $J$ for the above values of x, z, w, $H$ and $G$, using the iterative algorithm or solving eqns. 1-4.

7- Using a Mont Carlo simulation technique, a large sample is taken (6000points) by repeating steps 1-6.

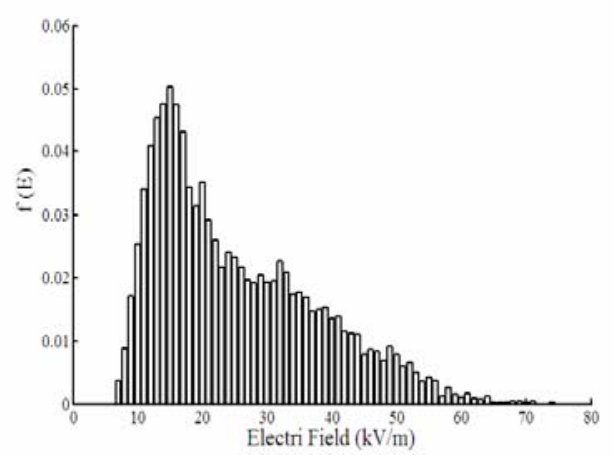

Fig.7 Histogram of $E$ - field, $w=0-2.5 \mathrm{~m} / \mathrm{s}$, $H=8.5-12.2 \mathrm{~m}, G=11.04-16.79 \mathrm{kV} / \mathrm{cm}$

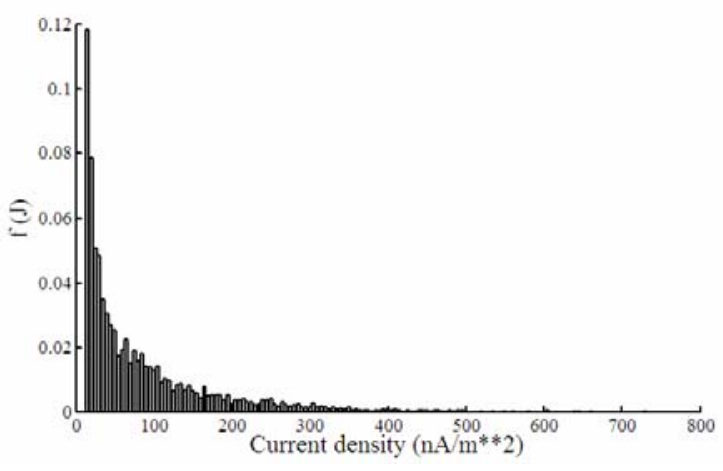

Fig.8 Histogram of $J$ - field, $w=0-2.5 \mathrm{~m} / \mathrm{s}$, $H=8.5-12.2 \mathrm{~m}, G=11.04-16.79 \mathrm{kV} / \mathrm{cm}$.

The probability density in this case, of a certain $E$ or $J$ value is the number of points where $E$ or $J$ attains that certain value relative to the total number of points. These relative points are computed and then used to construct the frequency of occurrence histogram, Figs. 7\&8 , of ambient field and current density values, due to the joint effect of all parameters. Using the above calculations, the maximum current intercepted by a $1.73 \mathrm{~m}$ person directly under the $4 \times 3.05 \mathrm{~cm}$ conductor operating at $600 \mathrm{KV}$ was calculated using the procedure in [5] and found to be $3.53 \mu \mathrm{A}$ in the downwind side. Correlation with the measured interception current of $3 \mu \mathrm{A}$ given in [5], is very good.

\section{CONCLUSIONS}

An integrated statistical method for calculating the electric field and the ion current density in the downwind side under the DC line has been presented, and the joint effect of wind, sag and corona onset gradient was simulated and established. The location of an object under the line has been taken uniformly random over the power line site and its influence upon the electric field and current density profiles was integrated into the analysis. Histograms of field and current density values near the line due to the random variation of each individual parameter were produced and a procedure has been devised to predict the probability of occurrence of $E$ and $J$ in each case. Correlation with measured values show good agreement. 


\section{REFERENCES}

[1] M. M. Abouelsaad, I.M. ElShair, I. Ghariany, "Numerical Assessment of Ionized Fields Under HVDC Lines”, The International Conference on Electrical, Electronic and Computer Engineering, ICEEC’ 4,2004, pp. 864-868.

[2]W.H. Bailey; D.E. Weil; J.R. Stewart "HVDC Power Transmission, Environmental Issues Review”, Technical Report ORNL/SUB-95-SR893/2, DOE Scientific and Technical Information, USA, April 1997.

[3] M. M. Abouelsaad, "A Probabilistic Model of Exposure to Ionized Fields Under HVDC Lines in Presence of Wind", IEEE Melecon 2002, May 2002,Cairo, Egypt, pp. 419-423.

[4] Xin Li, I.R. Ciric, and M.R. Raghuveer, "Investigation of Ionized Fields due to Bundled Unipolar DC Lines in the Presence of Wind", IEEE Trans. PWRD, Vol. 14, No. 1,1999, pp.211-217.

[5] H. L. Hill, A. S. Capon, O. Ratz, P. E. Renner, W. D. Schmidt, and Y. L. Miles, Transmission Line Reference Book-HVDC to $\pm 600 \mathrm{KV}, \mathrm{EPRI}, 3412$ Hillview Avenue, Palo Alto, CA 94304.

[6] P. S. Maruvada, R. D. Dallaire, O.C.Norris, C. V. Thio, and J. S. Goodman, "Environmental Effects Of The Nelson River HVDC transmission lines RI, AN, Electric Field, Induced Voltage, And Ion Current Distribution Tests" IEEE Trans., Vol. Pas-100, No. 4, April 1982, pp. 951-959.

[7]Y. Sunaga, V. Amano, and T. Sugimoto, " Electric field and ion current at the ground and voltage of charged objects under HVDC lines ", IEEE Trans. PAS, Vol.100, No. 4, 1981, pp. 2082-2092.

[8] H. L. Collins, R. G. Olsen, "HVDC Transmission Lines Generated Corona Behavior \& Characteristics" , A Report to the Bonneville Power Administration, DE-AI-85BP24408, Washington State University, 1988. 